\title{
Small-Angle X-ray Scattering Studies of Porcine Plasma Lipoproteins
}

\author{
By D. Atkinson and J. P. Green \\ Unilever Research Laboratory, Colworth/Welwyn, The Frythe, Welwyn, Herts., England
}

A high-density lipoprotein $\left(\mathrm{HDL}_{3}\right)$ from porcine plasma was studied by small-angle $\mathrm{X}$-ray scattering techniques. Experiments were conducted in the presence of different concentrations of sucrose, which was employed to raise the electron density of the solvent. The form of the scattering curves, and the well-resolved secondary maxima indicated a high degree of symmetry and/or particle homogeneity. Wide-angle studies confirmed the presence of highly mobile liquid-like hydrocarbon chains. The data allowed a calculation of the spherically-averaged electron-density distribution by Fourier inversion techniques. The range of experimentally determined scattering curves observed can be accounted for by a model for porcine $\mathrm{HDL}_{3}$ structure with the following features: a particle of radius of gyration $55 \AA$, comprising a central low electron density (lipid) core (radius $42 \AA$ ) and an outer electron dense polar annulus (thickness $12 \AA$ ). By combining the X-ray data, and results from other studies, the probable location of the constituent molecular species can be suggested.

J. Appl. Cryst. (1974). 7, 179

\section{X-ray Small-Angle Scattering of Human-Plasma Lipoproteins}

\author{
By Peter Laggner, Karl Müller and Otto Kratky \\ Institut für Röntgenfeinstrukturforschung, A-8010 Graz, Steyrergasse 17, Austria
}

The LpA fraction of human plasma high-density lipoprotein $\mathrm{HDL}_{3}$ has been studied by X-ray smallangle scattering in solvents of different electron densities using aqueous solutions of sucrose with different concentrations. The protein-to-lipid ratio of the preparation was 58:42 by weight. The molecular weight as determined by absolute scale measurements was $2 \cdot 1 \times 10^{5}$. In all solvents the scattering curves showed several well-resolved side maxima. In order to facilitate the analysis by Fourier transformation the intensities were extrapolated to zero in the region of the minima. The resulting radial electron density distributions were approximated by step functions convoluted with the experimental termination function. The experimental radii of gyration as a function of the solvent electron density served as a criterion for the decision between different models being equivalent in scattering. The model which was found to be consistent with the experimental results is a spherical particle with an outer diameter of $96 \AA$. An outer shell of $11 \AA$ thickness with an electron density of 0.386 e $\AA^{-3}$ surrounds a core of electron densities between 0.318 and $0.335 \mathrm{e} \AA^{-3}$. In terms of molecular structure this model allows for the surface location of the polypeptide chains and the polar phospholipid head groups. The $37 \AA$ radius of the low-electron-density core suggests a spherical micellar arrangement of the aliphatic chains of the phospholipids together with the cholesterol esters, which most probably are present in an extended conformation. Further the LpA fraction of human-plasma high-density lipoprotein $\mathrm{HDL}_{2}$ has been investigated. The method applied was in general quite similar to the one described above. The molecular weight amounts to $3 \cdot 7 \times 10^{5}$. The chemical analysis yielded a proteinto-lipid ratio of $42: 58$ by weight. The approximation of the radial electron density distribution by step models was carried out using a new computer program. Essentially the following model was found to be in best agreement with the experimental data: The molecule consists of two parts, an inner core of low electron density containing the main portion of the lipid moiety with a radius of $43 \AA$ and an outer shell of high electron density containing the polar groups of the phospholipids and the protein moiety. The total radius of the particle amounts to $57 \AA$. Finally measurements of human-plasma low-density lipoprotein LpB were performed. The evaluation of the experimental data is still in progress. results will be submitted shortly. 